\title{
Effect of Tetra-Alkyl Ammonium Bromide on the Rheological and Thermal Properties of Kappa-Carrageenan and Agarose Gels
}

\author{
Mineo Watase*, Katsuyoshi Nishinari, ${ }^{* *}{ }^{\dagger}$ Peter A. Williams, ${ }^{* *}$ \\ and Glyn O. PHILLIPs** \\ * Faculty of Liberal Arts, Shizuoka University, Ohya, Shizuoka 422, Japan \\ ** The North East Wales Institute of Higher Education, Deeside, Clwyd, CH5 4BR, UK
}

(Received April 16, 1990)

\begin{abstract}
Dynamic viscoelastic measurements and differential scanning calorimetry were carried out for kappa-carrageenan gels containing tetra-alkyl ammonium bromide in order to clarify the structure and functional properties of kappa-carrageenan. The dynamic Young's modulus $E^{\prime}$ of the gels showed a maximum as a function of concentration of tetra-alkyl ammonium bromide at around $0.2 \mathrm{~mol}^{-1}$. At any particular temperature $E_{\max }^{\prime}$ decreased in the following order: $E_{\max }^{\prime}\left(\mathrm{Me}_{4} \mathrm{~N}-\right)>E_{\max }^{\prime}\left(\mathrm{Et}_{4} \mathrm{~N}-\right)>E_{\max }^{\prime}\left(\operatorname{Pr}_{4} \mathrm{~N}-\right)>E_{\max }^{\prime}\left(\mathrm{Bu}_{4} \mathrm{~N}-\right)$. The temperatures $T_{m}$ of the endothermic peak accompanying the transition from gel to sol in the heating DSC curves shifted to higher temperatures with increasing salt concentration. The order of the melting temperature of these gels was the same as in $E_{\max }^{\prime}$, however, the enthalpy of melting showed the reverse order. Cations with smaller dynamic hydration number $n_{\mathrm{DHN}}$ increase the elastic modulus and the melting temperature of kappa-carrageenan gels more than those with larger $n_{\text {DHN }}$.

KEY WORDS Kappa-Carrageenan / Agarose / Gel / Tetra-Alkyl Ammonium Bromide / Rheology / Differential Scanning Calorimetry / Dynamic hydration Number /
\end{abstract}

Kappa-carrageenan and agarose are seaweed polysaccharides, and their chemical structures resemble each other: Kappa-carrageenan consists of D-galactose 4 sulfate and 3,6-anhydroD-galactose, while agarose consists of Dgalactose and 3,6-anhydro-L-galactose. However, their gelling properties are quite different: the gelling ability of kappa-carrageenan is increased by the addition of a small amount of alkali metal ions, alkali earth ions ${ }^{1-6}$ and guanidine hydrochloride. ${ }^{7}$ The addition of these chemical reagents in excess quantity decreases the gelling ability of kappa-carrageenan. The gelling ability of agarose is not influenced by alkali metal ions or alkali earth ions, but decreased by guanidine hydrochloride. The gelling mechanism of these polysaccharides is considered as follows: (i) single or double helices are formed from random coils by lowering the temperature of the solution, (ii) these helices aggregate to form an ordered structure which is called the junction zone or the crystalline region, and (iii) as a result, the three dimensional network is formed. Therefore, these gels consists of junction regions connected by long flexible chains. The junction zones are considered to be formed by hydrogen bonds. Kappa-carrageenan contains sulfate esters, while agarose does not. Therefore, the above mentioned experimental findings were understood as follows: The cations may shield the electrostatic repulsion between sulfate esters in kappacarrageenan molecules, and since the electro-

\footnotetext{
† Permanent Address: National Food Research Institute, Tsukuba 305, Japan.
} 
static repulsion inhibits the formation of helices of kappa-carrageenan molecules and their aggregation and tight binding, the introduction of cations promotes the aggregation of kappa-carrageenan molecules, and this increases the gelling ability. Since agarose has no sulfate esters, the cations have less influence on the gelling ability. This interpretation was confirmed by the effect of guanidine hydrochloride $(\mathrm{GuHCl})$ and urea on rheological and thermal properties of these gels. ${ }^{7}$ Both of these chemical reagents are hydrogen bond breakers. However, $\mathrm{GuHCl}$ is an electrolyte, and so the guanidinium ions shield the electrostatic repulsion between sulfate esters in kappacarrageenan as alkali metal ions or alkali earth metal ions do. Therefore, the addition of $\mathrm{GuHCl}$ below a certain concentration increases the gelling ability of kappa-carrageenan, but it decreases the gelling ability of agarose. Since urea is not an electrolyte, it only decreases the gelling ability of both polysaccharides.

Tetra-alkyl ammonium salts have attracted much attention from many investigators because of their importance in the study of hydrophobic interaction and the iceberg formation. $^{8}$

Grasdalen and Smidsrod ${ }^{2,9}$ reported that the conformational transition of kappa-carrageenan molecules detected by the change of specific optical rotation occurred by lowering the temperature in tetramethyl iodide but not in tetramethyl chloride. This result together with the invariance of molecular weights of tetramethyl-ammonium kappa-carrageenate in $\mathrm{N}\left(\mathrm{CH}_{3}\right)_{4}$ salts led them to the adoption of a single-stranded carrageenan-iodide complex rather than the double-helix structure that had previously been proposed by Morris and Rees. ${ }^{1}$ Norton et al. ${ }^{11}$ also observed the orderdisorder transition by optical rotation measurement for tetramethyl ammonium kappacarrageenate in the presence of $(\mathrm{Me})_{4} \mathrm{NCl}$, $(\mathrm{Me})_{4} \mathrm{NBr}$ and $(\mathrm{Me})_{4} \mathrm{NI}$. Although Grasdalen and Smidsrod $^{2,9}$ reported that this orderdisorder transition is unique to iodide, Norton et al. ${ }^{11}$ observed the analogous transitions in the presence of bromide and chloride coanions. The effect of alkali metal ions, ${ }^{12-14,18}$ and poly-hydric alcohols ${ }^{15}$ on the gel forming ability of kappa-carrageenan and agarose have been studied by rheological measurements and differential scanning calorimetry (DSC).

In the present work, the effects of tetra-alkyl ammonium salts on the rheological and thermal properties of Kappa-carrageenan and agarose gels were examined in order to compare with the effects of other chemical reagents on these properties. The gelling ability of kappacarrageenan will be influenced by two factors: (i) tetra-alkyl ammonium salts will play a role as the electrolyte and interact with kappacarrageenan molecules, and (ii) they will change the structure of water as a solvent and therefore will also affect the structure of the gels.

\section{EXPERIMENTAL}

\section{Materials}

Kappa-carrageenan was extracted from Chondrus crispus as used in the previous report. ${ }^{7}$ The intrinsic viscosity in $0.01 \mathrm{~N}$ $\mathrm{NaSCN}$ solution at $35^{\circ} \mathrm{C}$ was $14.0\left(100 \mathrm{ml} \mathrm{g}^{-1}\right)$. The potassium content in the specimen measured by atomic absorption spectrophotometry was $3.24 \%$. Agarose was extracted from Gelidium amansii as used in the previous report. ${ }^{7}$ It was pretreated with $3 \% \mathrm{NaOH}$, and then washed in flowing water. The intrinsic viscosity in $0.01 \mathrm{~N} \mathrm{NaSCN}$ at $35^{\circ} \mathrm{C}$ was 3.3 $\left(100 \mathrm{mlg}^{-1}\right)$. The preparation methods used in this study were the same as reported previously. ${ }^{7,12-15}$

Extra pure reagent grade $\left(\mathrm{CH}_{3}\right)_{4} \mathrm{NBr}$, $\left(\mathrm{C}_{2} \mathrm{H}_{5}\right)_{4} \mathrm{NBr},\left(\mathrm{C}_{3} \mathrm{H}_{7}\right)_{4} \mathrm{NBr}$ and $\left(\mathrm{C}_{4} \mathrm{H}_{9}\right)_{4} \mathrm{NBr}$ (Wako Pure Chemical Co., Ltd.) were used without further purification.

\section{Measurements}

The dynamic Young's modulus $E^{\prime}$ at $2.5 \mathrm{~Hz}$ was measured at various temperatures using a Rheolograph Gel CV-100 from Toyo Seiki 
Seisakusho Ltd. The temperature was controlled by a silicone oil bath, and the gel sample was kept at each measurement temperature for $20 \mathrm{~min}$. Differential scanning calorimetry (DSC) was carried out with a highly sensitive DSC SSC580 from Seiko Electronics Ltd. The heating rate was fixed as $2^{\circ} \mathrm{C} \mathrm{min}^{-1}$ throughout the present work. The details of these experimental methods were described previously. ${ }^{12-15}$

\section{RESULTS AND DISCUSSION}

Figures 1 and 2 show the dynamic Young's modulus $E^{\prime}$ of $2 \%$ w/w kappa-carrageenan gels as a function of concentration of tetra-alkyl
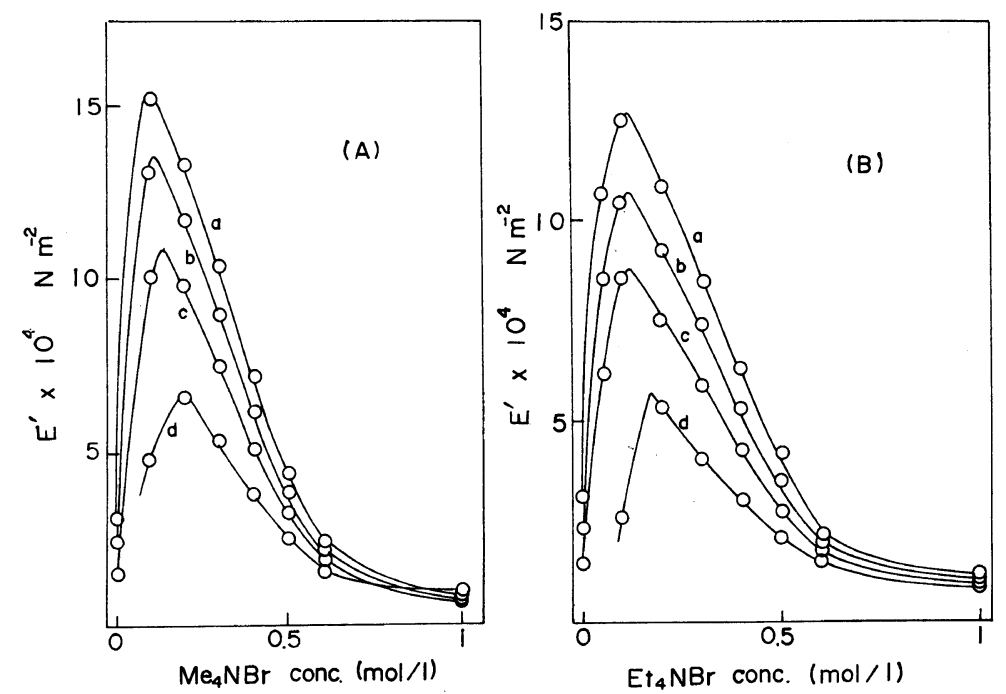

Figure 1. The dynamic Young's modulus $E^{\prime}$ of $2 \% \mathrm{w} / \mathrm{w}$ kappa-carrageenan gels with and without tetra-alkyl ammonium bromide as a function of concentration of $\mathrm{Me}_{4} \mathrm{NBr}(\mathrm{A})$ and $\mathrm{Et}_{4} \mathrm{NBr}(\mathrm{B})$ at various temperatures: $a, 15^{\circ} \mathrm{C} ; \mathrm{b}, 25^{\circ} \mathrm{C} ; \mathrm{c}, 35^{\circ} \mathrm{C} ; \mathrm{d}, 45^{\circ} \mathrm{C}$.
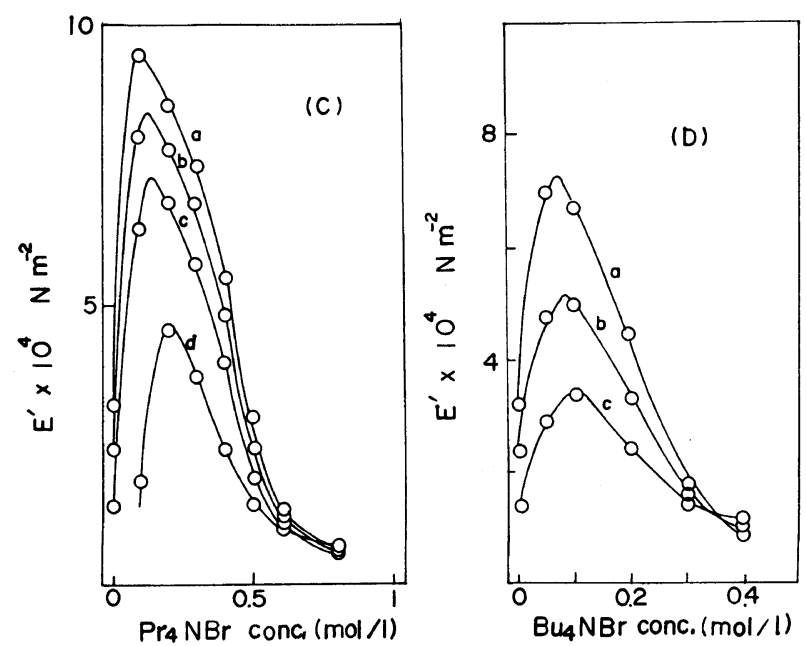

Figure 2. The dynamic Young's modulus $E^{\prime}$ of $2^{\circ} \mathrm{w} / \mathrm{w}$ Kappa-carrageenan gels with and without tetra-alkyl ammonium bromide as a function of concentration of $\operatorname{Pr}_{4} \mathrm{NBr}$ (C) and $n-\mathrm{Bu}_{4} \mathrm{NBr}$ (D) at various temperatures: $a, 15^{\circ} \mathrm{C} ; \mathrm{b}, 25^{\circ} \mathrm{C} ; \mathrm{c}, 35^{\circ} \mathrm{C} ; \mathrm{d}, 45^{\circ} \mathrm{C}$. 

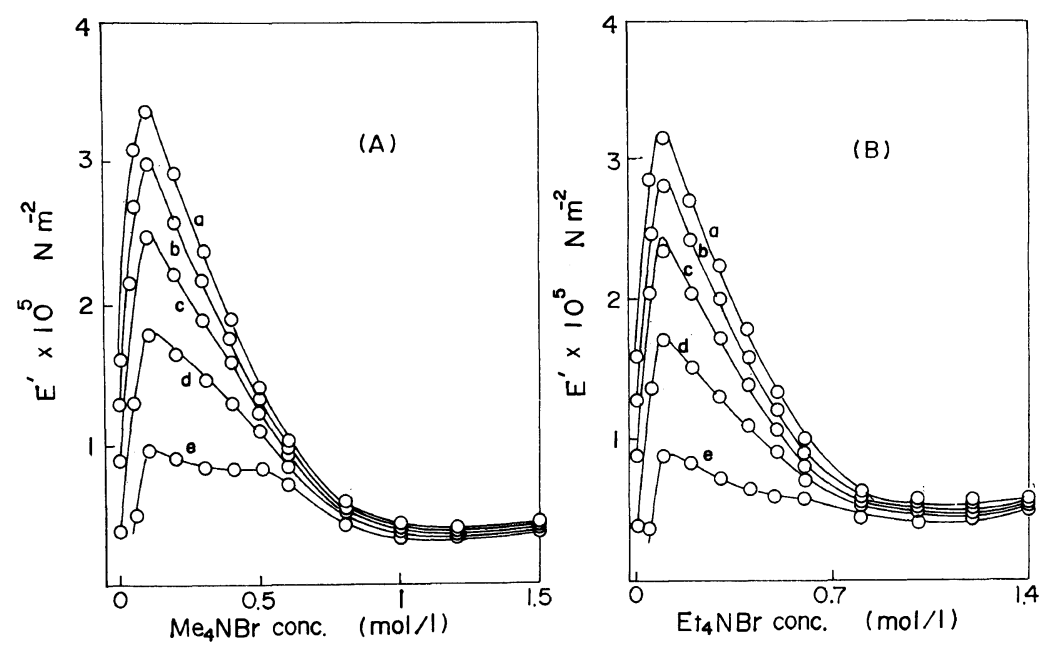

Figure 3. The dynamic Young's modulus $E^{\prime}$ of $3 \% \mathrm{w} / \mathrm{w}$ kappa-carrageenan gels with and without tetra-alkyl ammonium bromide as a function of concentration of $\mathrm{Me}_{4} \mathrm{NBr}(\mathrm{A})$ and $\mathrm{Et}_{4} \mathrm{NBr}(\mathrm{B})$ at various temperatures: $a, 15^{\circ} \mathrm{C} ; \mathrm{b}, 25^{\circ} \mathrm{C} ; \mathrm{c}, 35^{\circ} \mathrm{C} ; \mathrm{d}, 45^{\circ} \mathrm{C}, \mathrm{e}, 55^{\circ} \mathrm{C}$.
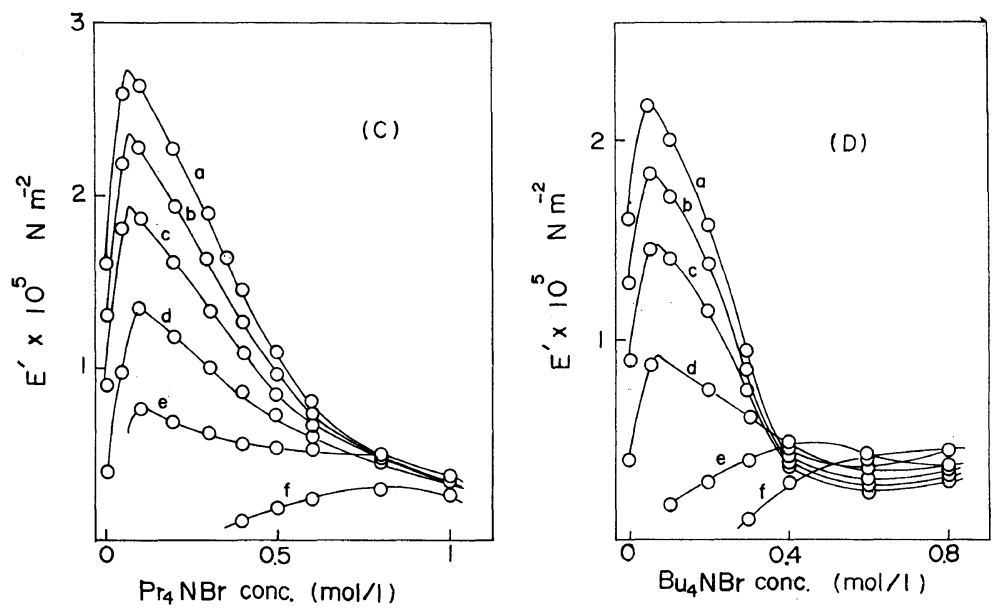

Figure 4. The dynamic Young's modulus $E^{\prime}$ of $3 \% \mathrm{w} / \mathrm{w}$ kappa-carrageenan gels with and without tetra-alkyl ammonium bromide as a function of concentration of $\operatorname{Pr}_{4} \mathrm{NBr}(\mathrm{C})$ and $n$ - $\mathrm{Bu}_{4} \mathrm{NBR}$ (D), at various temperature: $a, 15^{\circ} \mathrm{C} ; \mathrm{b}, 25^{\circ} \mathrm{C} ; \mathrm{c}, 35^{\circ} \mathrm{C}$;, $45^{\circ} \mathrm{C}$; e, $55^{\circ} \mathrm{C} ; \mathrm{f}, 65^{\circ} \mathrm{C}$.

ammionium bromide at various temperatures. $E^{\prime}$ increased sharply up to $0.1-0.2 \mathrm{~mol}^{-1}$ concentration of tetra-alkyl ammonium bromide and then decreased as the concentration increased further. The peak height of $E^{\prime}$ at the same temperature was in the following order: $E_{\max }^{\prime}\left(\mathrm{Me}_{4} \mathrm{~N}-\right)>E_{\max }^{\prime}\left(E_{4} \mathrm{~N}-\right)>E_{\max }^{\prime}\left(\operatorname{Pr}_{4} \mathrm{~N}-\right)>$ $E_{\max }^{\prime}\left(\mathrm{Bu}_{4} \mathrm{~N}-\right)$. The concentration of ammonium bromide at which $E^{\prime}$ becomes maximum shifted slightly to higher concentrations with increasing temperature.

Figures 3 and 4 shows the dynamic Young's modulus $E^{\prime}$ of $3 \%$ w/w kappa-carrageenan gels as a function of increasing concentration of tetra-alkyl ammonium bromide at various temperatures. General tendencies were the same as in $2 \%$ gels shown in Figures 1 and 2. However, the concentration of tetra-alkyl 


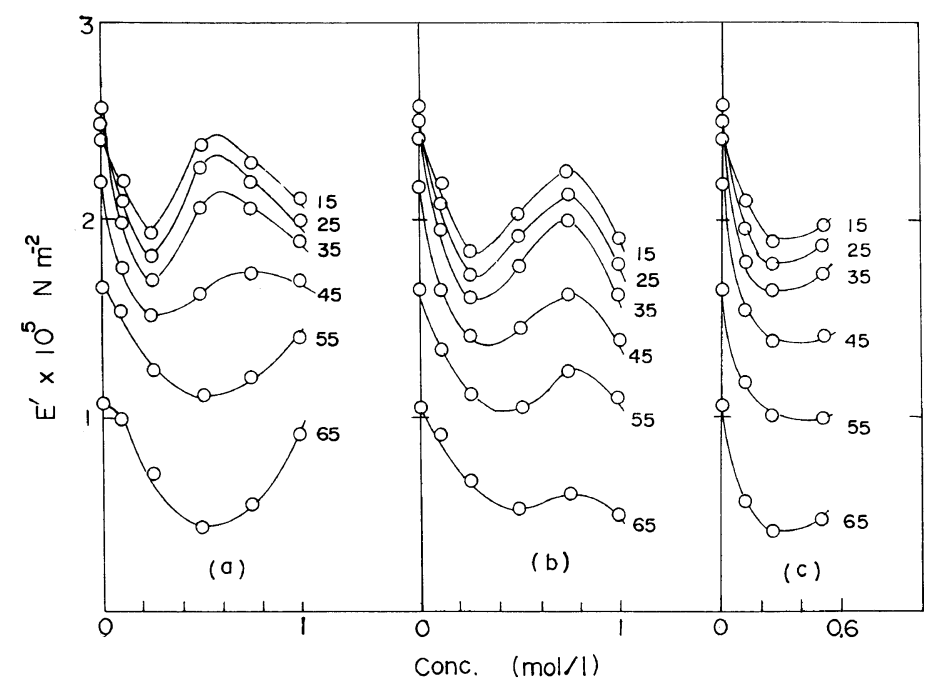

Figure 5. The dynamic Young's modulus $E^{\prime}$ of $2 \% \mathrm{w} / \mathrm{w}$ agarose gels with and without tetra-alklyl ammonium bromide as a function of concentration of $\mathrm{Me}_{4} \mathrm{NBr}(\mathrm{a}), \mathrm{Et}_{4} \mathrm{NBr}$ (b), and $n$ - $\mathrm{Bu}_{4} \mathrm{NBr}$ (c). Figures beside each curve represent the temperature in ${ }^{\circ} \mathrm{C}$.

ammonium bromide at which $E^{\prime}$ becomes maximum shifted remarkably to higher concentrations with increasing temperature in gels containing $\mathrm{Pr}_{4} \mathrm{NBr}$ (Figure 4 (C)) and $\mathrm{Bu}_{4} \mathrm{NBr}$ (Figure 4 (D)).

Figure 5 shows the dynamic Young's modulus $E^{\prime}$ of $2 \%$ w/w agarose gels containing tetra-alkyl ammonium bromide as a function of the concentration of tetra-alkyl ammonium bromide. The change of $E^{\prime}$ induced by tetra-alkyl ammonium bromide was smaller in comparison to kappa-carrageenan gels. The values of $E^{\prime}$ for agarose gels scarcely changed by the introduction of alkali metal salts ${ }^{12,13}$ because agarose does not contain any groups which are influenced by salts. However, the change of $E^{\prime}$ for agarose gels induced by tetra-alkyl ammonium bromide is not negligible. $E^{\prime}$ of $2 \% \mathrm{w} / \mathrm{w}$ agarose gels as a function of the concentration showed a minimum around $0.2-0.5 \mathrm{~mol}^{-1}$ concentration of tetra-alkyl ammonium bromide and this was followed by a maximum in gels containing $\mathrm{Me}_{4} \mathrm{NBr}$ and $\mathrm{Et}_{4} \mathrm{NBr}$.

Figures 6 and 7 show DSC heating curves of $2 \% \mathrm{w} / \mathrm{w}$ kappa-carrageenan gels with and without tetra-alkyl ammonium bromide. An endothermic peak accompanying the transition from gel to sol was observed in all cases. The peak temperature $T_{\mathrm{m}}$ shifted to higher temperatures with increasing concentration of tetra-alkyl ammonium bromide. Norton et $a l .{ }^{11}$ found that the order-disorder transition temperature of kappa-carrageenan gels shifted to higher temperatures with increasing tetramethyl ammonium bromide by optical rotation measurements, which corresponds well to our results shown in Figure 6(A). This shift was most marked in gels containing $\mathrm{Me}_{4} \mathrm{NBr}$, and then in the order $\mathrm{Et}_{4} \mathrm{NBr}, \mathrm{Pr}_{4} \mathrm{NBr}, \mathrm{Bu}_{4} \mathrm{NBr}$, i.e., this shift became less marked in the order of the size of cations. It seems that too bulky cations are not as effective in promoting the formation of junction zones.

The DSC endothermic peak became sharper up to a certain concentration of tetra-alkyl ammonium bromide and then became broader beyond that concentration with increasing concentration of the salt. This corresponds well to the rheological finding that $E^{\prime}$ increased up to a certain concentration of tetraalkyl ammonium bromide and then began to 

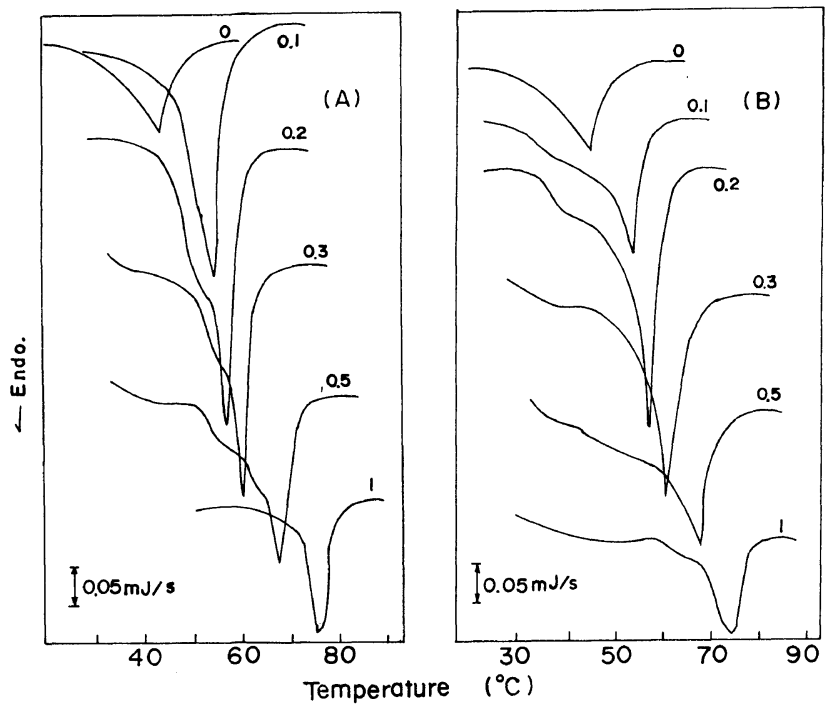

Figure 6. Heating DSC curves of $2 \% \mathrm{w} / \mathrm{w}$ kappa-carrageenan gels with and without tetra-alkyl ammonium bromide. Figures beside each curve represent the concentration of $\mathrm{Me}_{4} \mathrm{NBr}(\mathrm{A})$ and $\mathrm{Et}_{4} \mathrm{NBr}$ (B) in $\mathrm{moll}^{-1}$.
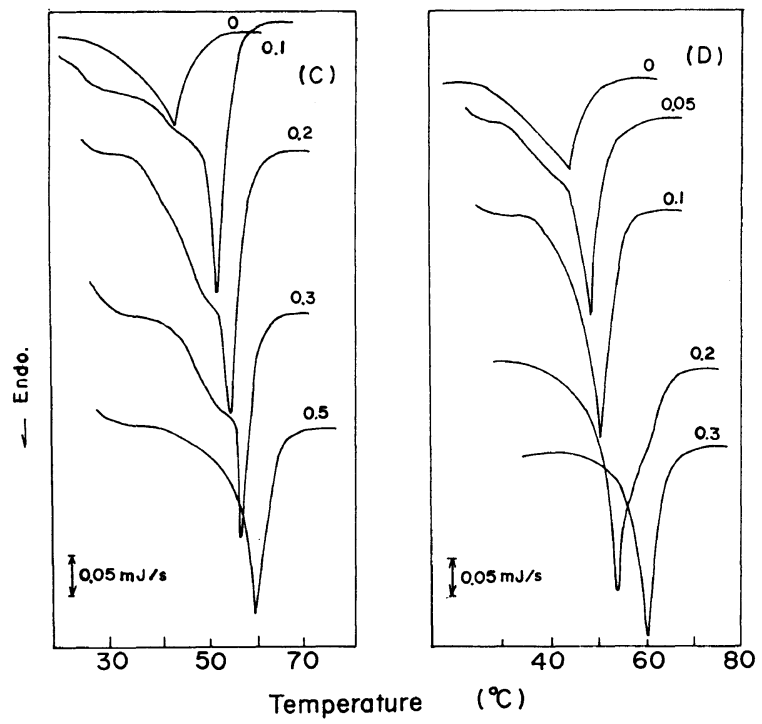

Figure 7. Heating DSC curves of $2 \% \mathrm{w} / \mathrm{w}$ kappa-carrageenan gels with and without tetra-alkyl ammonium bromide. Figures beside each curve represent the concentration of $\mathrm{Pr}_{4} \mathrm{NBr}(\mathrm{C})$ and $n-\mathrm{Bu}_{4} \mathrm{NBr}$ (D) in $\mathrm{mol} \mathrm{l}^{-1}$.

decrease with increasing concentration of the salt. This tendency has also been observed in kappa-carrageenan gels containing alkali metal salt, alkali earth metal salt, and guani- dine hydrochloride. The appearance of gels changes from solid-like or rubber-like to liquid-like beyond a certain concentration of the added chemical reagents. Since the DSC 
endothermic peak is equivalent to the maximum of heat capacity, the gel-sol transition was explained recently by a zipper model approach. ${ }^{16}$ The approach is based on a model dealing with the melting of thermoreversible gels whose junction zones mainly consist of crystalline regions resulting of association of rigid ordered molecular structure such as helices or extended rod-like molecules. A zipper is here a dimer of macromolecular chains formed by parallel links. According to this treatment, the DSC endothermic peak is determined mainly by the bonding energy $E$ which makes aggregate the ordered molecules such as double helices or extended rigid molecules, by the rotational freedom $\mathrm{G}$ of links around the aggregate, and by the number of bonds $\mathrm{N}$. When the experimental DSC endotherm is sharp as in the case of kappa-carrageenan without any chemical reagent, it is well reproduced by one set of $\mathrm{E}, \mathrm{G}$, and $\mathrm{N}$. However, introduction of tetra-alkyl ammonium bromide promotes the aggregation of helical kappa-carrageenan molecules, and so the gel tends to a more ordered structure. However, excessive salt immobilises free water which is necessary for junction zone formation, or hinders the aggregation of helices although the junction zone itself seems to become more heat-resistant. At the same time, the excessive salt seems to induce the distribution of $\mathrm{E}, \mathrm{G}$, and $\mathrm{N}$. Therefore, the DSC endothermic peak becomes broader in the presence of excessive salt. This situation may be represented in Figure 8 .

Figure 9 shows the plot of $\log C$ against $1 / T_{\mathrm{m}}$ for carrageenan gels, where $C$ is the concentration of the salt. According to Manning's theory, ${ }^{17}$ this plot will become a straight line at lower concentrations, and the slope of the straight line obtained from this plot is proportional to the enthalpy $\Delta H$ of melting of the gels. As is seen from Figure 9, the plot deviates at higher concentrations, and the magnitude of $\Delta H$ was in the following order: $\Delta H\left(\mathrm{Bu}_{4} \mathrm{~N}-\right)>\Delta H\left(\mathrm{Pr}_{4} \mathrm{~N}-\right)>\Delta H\left(\mathrm{Et}_{4} \mathrm{~N}-\right)>$

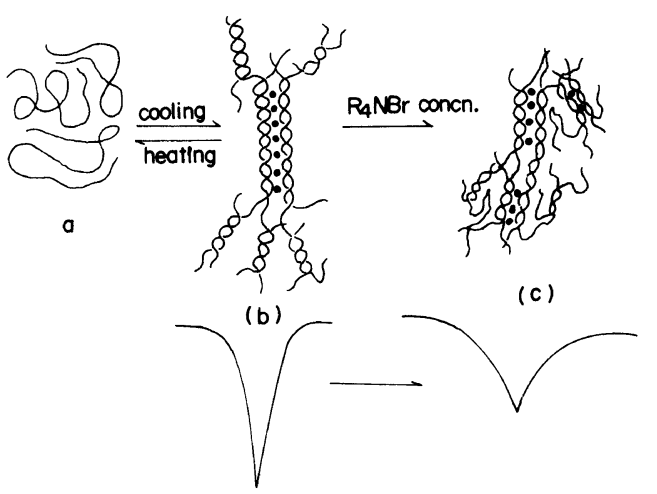

Figure 8. The gelation mechanism of kappa-carrageenan. Filled circles represent the tetra-alkyl ammonium ions. (a), random coil: (b), junction zone; (c), junction zone. (b) corresponds to a sharp DSC endothermic peak, while (c) corresponds to a broad DSC endothermic peak.

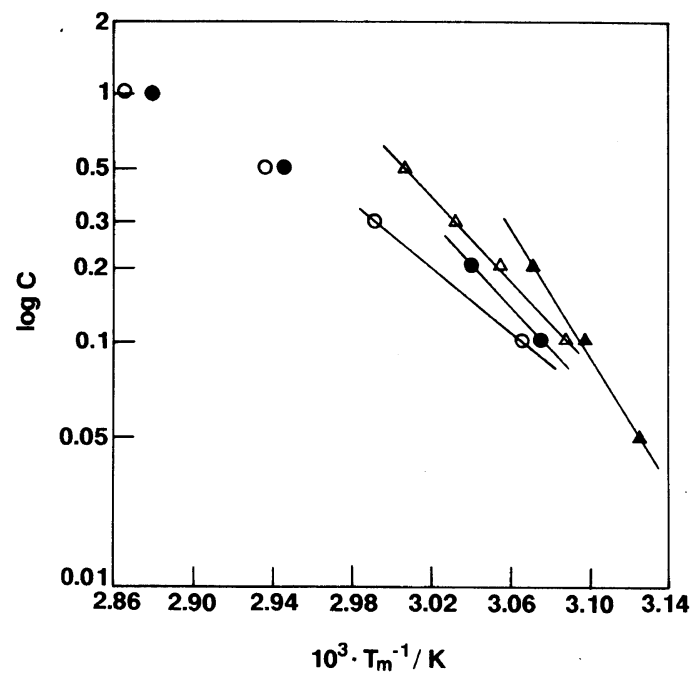

Figure 9. The relation between the inverse of melting temperature $T_{\mathrm{m}}$ of $2 \% \mathrm{w} / \mathrm{w}$ kappa-carrageenan gels containing tetra-alkyl ammonium bromide and the logarithm of the concentration of tetra-alkyl ammonium bromide: $\bigcirc, \mathrm{Me}_{4} \mathrm{NBr} ; \bigcirc, \mathrm{Et}_{4} \mathrm{NBr} ; \triangle, n-\mathrm{Pr}_{4} \mathrm{NBr} ; \boldsymbol{\Delta}$ $n-\mathrm{Bu}_{4} \mathrm{NBr}$.

$\Delta H\left(\mathrm{Me}_{4} \mathrm{~N}-\right)$, where $\Delta H\left(\mathrm{Bu}_{4} \mathrm{~N}-\right)$ represents the enthalpy of metling of gels containing $\mathrm{Bu}_{4} \mathrm{NBr}$. Gels formed in the presence of $\mathrm{Bu}_{4} \mathrm{NBr}$, therefore, have the lowest value for $E_{\max }^{\prime}$ and $T_{\mathrm{m}^{\prime}}$ but the highest enthalpy of melting. This behaviour is contrary to that observed generally for polymer gels ${ }^{18,19}$ and further 


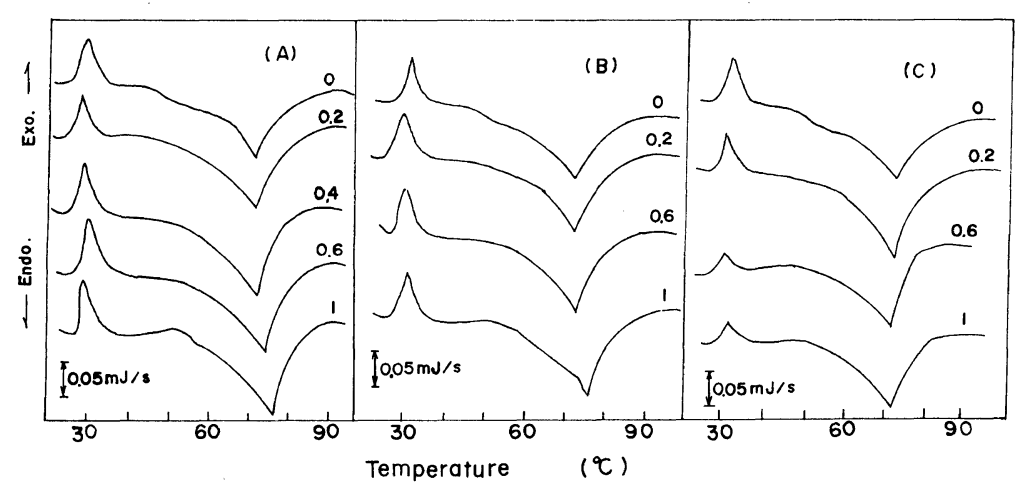

Figure 10. Heating DSC curves of $2 \% \mathrm{w} / \mathrm{w}$ agarose gels with and without tetra-alkyl ammonium bromide. Figures beside each curve represent the concentration of $\mathrm{Me}_{4} \mathrm{NBr}(\mathrm{A}), \mathrm{Et}_{4} \mathrm{NBr}(\mathrm{B})$, and $n-\mathrm{Bu}_{4} \mathrm{NBr}(\mathrm{C})$ in $\mathrm{mol} \mathrm{l}^{-1}$.

work is required in order to fully explain the experimental results.

Figure 10 shows the heating DSC curves of $2 \% \mathrm{w} / \mathrm{w}$ agarose gels with and without tetra-alkyl ammonium bromide. The small exothermic peaks which appeared at about $30^{\circ} \mathrm{C}$ were supposed to be induced by molecular rearrangement into a more ordered structure during heating, which accompanies syneresis. These exothermic peaks appear only in the first heating run, and disappear in the reheating DSC curves. The endothermic peak accompanying the transition from gel to sol shifted slightly to higher temperatures in the case of $\mathrm{Me}_{4} \mathrm{NBr}$ and $\mathrm{Et}_{4} \mathrm{NBr}$, while it shifted to slightly lower temperatures in the case of $\mathrm{Bu}_{4} \mathrm{NBr}$.

Figure 11 shows the plot of the value $\mathrm{d} T_{\mathrm{m}} / \mathrm{d} \log C$ taken from Figure 9 against the dynamic hydration number $n_{\mathrm{DHN}}$, where $n_{\mathrm{DHN}}$ is defined as $n_{\mathrm{h}}\left(\tau_{\mathrm{c}}{ }^{\mathrm{h}} / \tau_{\mathrm{c}}{ }^{0}-1\right){ }^{20-22}$ Here, $n_{\mathrm{h}}$ is the coordination number, $\tau_{\mathrm{c}}^{\mathrm{h}}$ is the rotational correlation time of water molecules around the ammonium salts, and $\tau_{\mathrm{c}}{ }^{0}$ is the rotational correlation time of pure water. The dynamic hydration number of the ammonium ion is estimated as -0.55 by Uedaira. ${ }^{23}$ The melting temperature decreased with increasing the dynamic hydration number of cations. It is well known that cations such as alkali metal ions and alkali earth metal ions enhance the gelling

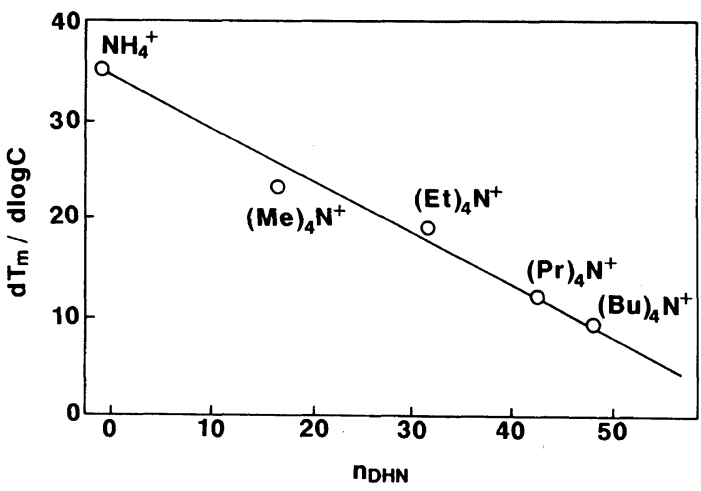

Figure 11. Plot of $\mathrm{d} T_{\mathrm{m}} / \mathrm{d} \log C$ ( $C$, concentration of tetra-alkyl ammonium bromide) for $2 \% \mathrm{w} / \mathrm{w}$ kappacarrageenan gels containing tetra-alkyl ammonium bromide as a function of the dynamic hydration number $n_{\mathrm{DHN}}$ of cations.

ability of kappa-carrageenan molecules. ${ }^{1-6}$ If the electrostatic repulsion of sulfate groups in kappa-carrageenan molecules is suppressed by cations, the formation of helices and their aggregation is promoted, and hence the gelling ability is increased. It was found that $\mathrm{K}^{+}$and $\mathrm{Cs}^{+}$are more effective is increasing the elastic modulus and shift the melting temperature $T_{\mathrm{m}}$ to higher temperatures for kappa-carrageenan gels than $\mathrm{Li}^{+}$and $\mathrm{Na}^{+} .3,12,13$ The reason was attributed to the fact that structure breaking ions such as $\mathrm{K}^{+}$and $\mathrm{Cs}^{+}$shield the electrostatic repulsion of sulfate groups more effectively 
than structure making ions such as $\mathrm{Li}^{+}$and $\mathrm{Na}^{+}$because electrostatic shielding of structure making ions is weakened by intervening water molecules. The concept of dynamic hydration number $n_{\mathrm{DHN}}$ is more quantitative than that of structure making or breaking ions; larger $n_{\mathrm{DHN}}$ indicates structure making, while smaller $n_{\mathrm{DHN}}$ indicates structure breaking. Therefore, the relation between $n_{\mathrm{DHN}}$ of tetra-alkyl ammonium ions and $\mathrm{d} T_{\mathrm{m}} / \mathrm{d} \log C$ shown in Figure 11 can be understood just as in the case where alkali metal ions are added to kappacarrageenan solution and to increase the gelling ability. ${ }^{3,12,13}$ Cations with smaller dynamic hydration number $n_{\text {DHN }}$ increase the elastic modulus and the melting temperature of kappa-carrageenan gels than those with larger $n_{\text {DHN }}$.

Acknowledgements. Authors thank Prof. $\mathrm{H}$. Uedaira for valuable discussions on dynamic hydration number.

\section{REFERENCES}

1. E. R. Morris, D. A. Rees, and G. J. Robinson, Mol. Biol., 138, 349 (1980).

2. O. Smidsrod and H. Grasdalen, Hydrobiologia, 116/117, 19 (1984).

3. M. Watase and K. Nishinari, J. Texture Stud., 12, 427 (1981).

4. V. J. Morris and G. R. Chilvers, Carbohydr. Polym., 3, 129 (1983).

5. R. Rinaudo and C. Rochas, in "Solution Properties of Polysaccharides," D. A. Brant, Ed., Amer. Chem. Soc. Symp. Series 150, Washington D. C. American Chemical Society, 1981, p. 367.

6. D. Day, G. O. Phillips, and P. A. Williams, Food Hydrocolloids, 2, 19 (1988).

7. M. Watase and K. Nishinari, Food Hydrocolloids, 1, 25 (1986).

8. W. Y. Wen, in "Water and Aqueous Solution," R. A. Horne, Ed., Wiley, New York, 1972, Chapter 15.

9. H. Grasdalen and O. Smidsrod, Macromolecules, 14, 1842 (1981).

10. D. A. Rees, E. R. Morris, D. Thom, and J. K. Madden, in "The Polysaccharides," for G. O. Aspinall, Ed., Academic Press, New York, 1982, Chapter 5.

11. I. T. Norton, E. R. Morris, and D. A. Rees, Carbohydr. Res., 194, 89 (1984).

12. M. Watase and K. Nishinari, Rheol. Acta, 21, 318 (1982).

13. M. Watase and K. Nishinari, Colloid Polym. Sci., 260, 971 (1982).

14. M. Watase and K. Nishinari, Polymer J., 18, 1017 (1986).

15. K. Nishinari and M. Watase, Agric. Biol. Chem., 51, 3231 (1987).

16. K. Nishinari, S. Koide, P. A. Williams, and G. O. Phillips, J. Phys. (France), 51, 1759 (1990).

17. G. S. Manning, Biopolymers, 11937 (1972).

18. M. Watase and K. Nishinari, Makromol. Chem., 188, 2213 (1987).

19. H. M. Tan, A. Moet, A. Hiltner, and E. Baer, Macromolecules, 16, 28 (1983).

20. H. Uedaira, M. Ikura, and H. Uedaira, Bull. Chem. Soc. Jpn., 62, 1 (1989).

21. M. Ishimura and H. Uedaira, Bull. Chem. Soc. Jpn., 63, 1 (1990).

22. H. Uedaira, "Hydrogels and Water," in "The Science of Food Hydrocolloids," K. Nishinari and T. Yano, Ed., Asakura Shoten, Tokyo, (1990).

23. H. Uedaira, private communication (Sapporo). 